\title{
Removal of phase transfer agent leads to restricted dynamics of alkyl chains in monolayer protected clusters ${ }^{\dagger}$
}

\author{
V R RAJEEV KUMAR ${ }^{1}$, R MUKHOPADHYAY ${ }^{2}$ and T PRADEEP ${ }^{1, *}$ \\ ${ }^{1}$ DST Unit on Nanoscience, Department of Chemistry and Sophisticated Analytical Instrument Facility, \\ Indian Institute of Technology Madras, Chennai 600036 \\ ${ }^{2}$ Solid State Physics Division, Bhabha Atomic Research Centre, Trombay, Mumbai 400085 \\ e-mail: pradeep aiitm.ac.in
}

\begin{abstract}
The effect of phase transfer agent in the dynamics of monolayer protected gold nanoparticles has been investigated by infrared (IR) and nuclear magnetic resonance (NMR) spectroscopies. The experiments were performed with octadecane thiol and dodecane thiol protected gold nanoparticles. The materials prepared were characterized by UV-Visible spectroscopy, transmission electron microscopy and IR spectroscopy. Repeated purification of the monolayer protected gold clusters made the alkyl chains defect-free. Such effects are reflected in the infrared spectra. Interdigitation of the monolayers that followed the purification leads to alkyl chains with limited mobility. This was reflected in ${ }^{13} \mathrm{C}$ and ${ }^{1} \mathrm{H}$ NMR linewidths. The NMR measurements indicate that the removal of phase transfer agent affects the dynamics of isolated clusters and those with interdigitated monolayers in different ways.
\end{abstract}

Keywords. Monolayer protected clusters; dynamics; phase transfer agent; thiols; nanoparticles.

\section{Introduction}

Alkane thiols on metal surfaces are model systems to study many fundamental problems. Studies of monolayers by bulk techniques were limited because of the unavailability of materials in the powder form. In 1994, Schriffin et al reported a biphasic reduction method to prepare metal nanoparticles in high yield ${ }^{1}$. The solution phase route using phase transfer catalysis to prepare three-dimensional self assembled monolayers (3D SAMs) allowed detailed studies of these systems with various spectroscopic and microscopic methods. Murray called such nanoparticles as monolayer protected clusters (MPCs). ${ }^{2}$ Infrared spectroscopic studies proved that the structure of alkane thiols on 3D SAMs parallel in many aspects alkane thiols on planar surfaces (2D SAMs). ${ }^{3-4}$ A remarkable feature was the minimum number of gauche defects near the head group for the 3D SAMs similar to 2D SAMs, even though nanocrystals offered various facets for the alkane thiol to exhibit gauche defects. The only difference was the larger concentration of chain end gauche defects. The hydrodynamic radius calculated from the electrochemical measurements was less when compared to that calculated by taking the sum of the gold core radius and the

\footnotetext{
${ }^{\dagger}$ Dedicated to Prof. C N R Rao on his 75th birthday

*For correspondence
}

length of the alkyl chain, assuming the chain to be in an all-trans extended conformation. This indicates the possibility of lateral chain folding in MPCs. ${ }^{5}$

The spin-lattice relaxation measurements indicated the existence of carbons with varying mobilities along the chain. ${ }^{6}$ The decay of the all-trans methylene peak could be fit into a biexponential curve with a long-lived component corresponding to the interior methylenes near the bound end and a short-lived component corresponding to the mobile carbons at the chain end. The relaxation time of terminal methyl group was of the order of seconds indicating that they are freely rotating. A comparison of the methylene carbon relaxation times of methyl and hydroxyl terminated 3D SAMs indicated that the methylene chain motion is more restricted in hydroxyl terminated SAMs. ${ }^{7}$

A more detailed dynamic view of the monolayer was available from the dynamic dephasing experiments. ${ }^{6}$ These studies proved that the skin of the monolayer exhibits considerable gauche defects whereas the carbons towards the interior were alltrans and highly crystalline. The interior carbons were in a restricted state whereas the outermost carbons were highly mobile and conformationally disordered. Such all-trans highly crystalline, wellpacked interior carbons prevented the dynamics, resulting in large relaxation times. 
Temperature dependent infrared spectroscopic studies on alkane thiols, deuterated at specific positions, showed that melting starts from the chain terminus and it propagates to the middle of the chain upon increasing the temperature. ${ }^{8}$ Even at high temperatures, the population of gauche defects present near the sulfur head group was minimum. Molecular dynamics (MD) simulations of monolayers on planar surfaces also revealed that majority of the defects are located at the chain terminus whereas the middle of the chain was defect-free. ${ }^{9}$ On increasing the temperature, MD simulations showed that the population of gauche defects started increasing in the middle but could not propagate towards the head group. This again supports the fact that $3 \mathrm{D}$ SAMs parallel in many aspects monolayers on planar surfaces.

The effect of core size on the dynamics of alkyl chain was studied by Murray et al. ${ }^{10}$ They prepared dodecane thiol protected gold nanoparticles with core sizes varying from 0.76 to $2.6 \mathrm{~nm}$. The line width in the ${ }^{13} \mathrm{C}$ spectrum was found to increase with decrease in metal core diameter. Since the alkyl chain was the same for all the samples, the change in linewidth was attributed to the change in the core diameter. They also pointed out the importance of spin-spin relaxation broadening $\left(T_{2}\right)$ in NMR measurements. They found that the width of the methyl resonance changes systematically with distance from the metal core.

We have been interested in the dynamics of alkane thiol protected gold clusters for some time. ${ }^{11}$ In one of the reports, we compared the dynamics of octadecane thiol protected gold clusters and octane thiol protected silver nanoparticles with a model planar 2D system, namely, layered silver thiolate. ${ }^{12}$ Octane thiol protected silver nanoparticle was selected because from our own previous studies, we know that these monolayers show high propensity to form superlattices. ${ }^{13}$ We found that dynamics starts much early in isolated clusters compared to superlattices. The dynamics observed in the superlattice and thiolates were very similar. We proposed that all the systems exhibited uniaxial rotational chain diffusion about the axis. We extended the studies to gold nanoparticles protected with hexane, dodecane and octadecane thiols. ${ }^{14}$ The analysis of elastic incoherent structure factor (EISF) revealed that only a few percentage of the total chain is dynamic at low temperature. The dynamics spread to all other chains on increasing the temperature to $400 \mathrm{~K}$. Based on the experimental data, we proposed that alkyl chains undergo simultaneous uniaxial rotational motion and body axis fluctuation.

The major drawback of the solution phase route using phase transfer catalysis to prepare the SAMs is the difficulty of removing the adsorbed phase transfer reagent (PTR) as this would affect the properties of the final SAMs. The presence of PTR in the final material has been confirmed by a number of studies. Our own studies using laser desorption ionization (LDI) mass spectrometry supported the fact that the presence of PTR has influence on the dynamics of the material. ${ }^{14}$

Although much is known about the dynamics of alkyl chains on metal nanoparticles, a systematic study of the effect of PTR on the dynamics of the alkyl chain has not yet been performed. This is important as the dynamics may be hidden by the presence of PTR coexisting with the alkane thiols. In this report, we show that the order present in the alkyl chain is considerably improved upon repeated purification. However, repeated PTR removal increased the interdigitation between the chains which prevented the alkyl chains from exhibiting dynamics. This is reflected in the width of certain carbon resonances as well as the proton resonances in NMR spectroscopy.

\section{Experimental}

\subsection{Chemicals}

Hexane thiol, dodecane thiol and sodium borohydride were purchased from Sigma Aldrich. Tetraoctylammonium bromide (TOAB) was purchased from Merk. Toluene was distilled prior to use.

\subsection{Synthesis}

The thiol protected gold nanoparticles used in the study were prepared following the method reported by Schffriin et al. ${ }^{1} 30 \mathrm{~mL} \mathrm{HAuCl}_{4}$ solution $(30 \mathrm{mM})$ was mixed with $80 \mathrm{~mL}$ TOAB solution $(50 \mathrm{mM})$. Vigorous stirring of the solution resulted in the phase transfer of $\mathrm{AuCl}_{4}^{-}$. After the phase transfer, the aqueous layer was discarded. $0.840 \mathrm{~mol}$ of neat octadecane thiol was added to the organic phase. The aurothiolate in the organic phase was reduced by drop-wise addition of $25 \mathrm{~mL}$ freshly prepared aqueous $\mathrm{NaBH}_{4}$ solution $(0.4 \mathrm{M})$. The organic phase was separated and precipitation with isopropyl alcohol 
gave particles with average diameter in the range 2 $3 \mathrm{~nm}$. The studies were carried out with dodecane thiol protected gold nanoparticles $(\mathrm{Au} @ \mathrm{C} 12)$ and octadecane thiol capped gold nanoparticles $(\mathrm{Au} @ \mathrm{C} 18)$.

\subsection{Purification}

The monolayer protected gold nanoparticles prepared by the Schriffin protocol were purified by precipitating from toluene/isopropyl alcohol mixture. The precipitation was done once, twice and thrice to see the effect of phase transfer agent on the dynamics of the alkyl chain.

\subsection{Instrumentation}

UV-Visible spectra were recorded with a Perkin Elmer Lambda 25 spectrometer. Transmission electron microscopic images were taken using a JEOL 3010 high resolution transmission electron microscope (HRTEM) operating at $300 \mathrm{keV}$. Specimens for the measurements were prepared by spreading a small drop of a dilute dispersion of the materials on a 200 mesh carbon coated copper grid. FT-IR measurements were done with a Perkin Elmer Spectrum one spectrometer. The specimen was prepared as a $\mathrm{KBr}$ pellet of the dry material. The NMR measurements were carried out on a GEL GSX 400 NMR spectrometer.

\section{Results and discussion}

Figure 1 shows the UV-Vis spectrum of the material after each stage of purification. Curve $a$ is before purification. Curves $b, c$, and $d$ represent the UVVis spectra after precipitating once, twice and thrice from toluene-isopropyl alcohol mixture. The surface plasmon peak, due to the collective oscillation of conduction band electrons, was present in all the samples. The shape of the spectrum is characteristic of particles in this size range. ${ }^{13}$ The peak position was the same for all samples except that the background was rising continuously, especially in curve $d$. The increase in background is due to the formation of aggregates consequent to the interdigitation of the alkyl chains, as a result of the removal of PTR. The TEM measurements clearly show that the core size is invariant during purification and no specific aggregate structures were observed. The average inter-particle distance in image $d$ is smaller than the other three images (see figure 2). Thus, increased background indicates that the monolayers of adjacent nanoparticles are indeed interdigitating. Such interdigitated particles contribute to enhanced scattering. The increase in background in the longer wavelength region, due to the interdigitation of alkyl chains leading to the formation of superlattices, is reported in the literature. ${ }^{15}$ This increased background disappears when the superlattice dissolves to form isolated particles.

High resolution TEM images of Au@C18 after successive precipitation are shown in figure 2. The images show that the average core size remained invariant throughout the purification, for all the materials. Many of the particles showed lattice fringes (marked with arrows). The distance between the particles was in the range of $1 \mathrm{~nm}$, indicating interdigitation of the alkyl chains. After the third purification, the particle showed higher propensity to form interdigitated structures. This indicates that although the alkyl chain is defect free, PTR present in the initial stage prevents the particles from interacting with each other.

Figure $3 \mathrm{~A}$ shows the IR spectrum in the range of $2700-3050 \mathrm{~cm}^{-1}$ for $\mathrm{Au} @ \mathrm{C} 18$ after each stage of purification. The positions of the methylene symmetric stretching $\left(d^{+}\right)$and antisymmetric stretching $\left(d^{\top}\right)$ peaks indicate the order present in the alkyl chains. For crystalline alkyl chains, the $\left(d^{+}\right)$and $\left(d^{-}\right)$peaks appear at 2850 and $2920 \mathrm{~cm}^{-1} \cdot{ }^{16-17}$ In the case of liquid-like alkane, the peak shifts towards high energies.

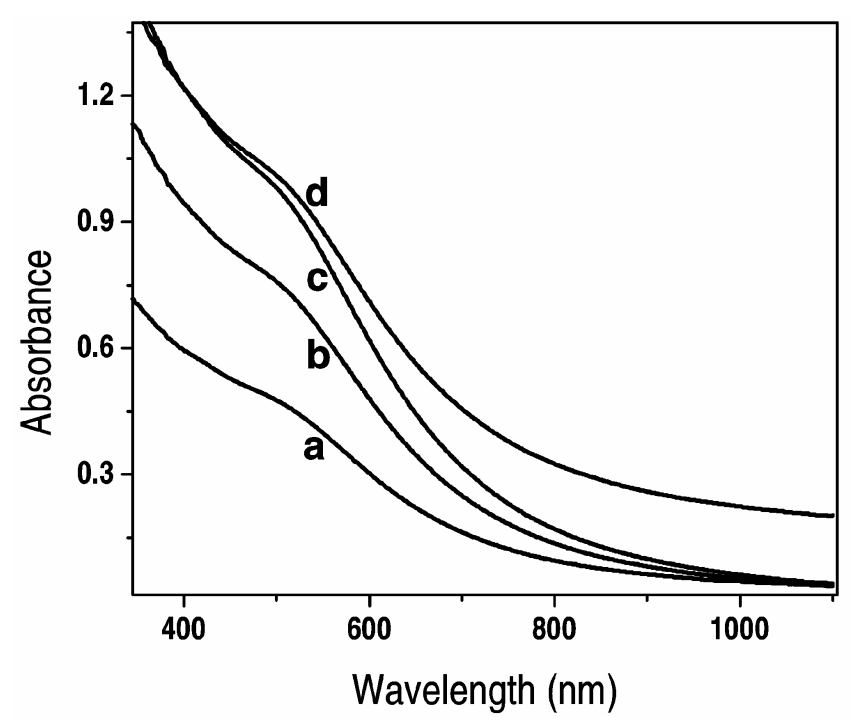

Figure 1. UV-Vis spectra of $\mathrm{Au} @ \mathrm{C} 18$. (a) Before precipitation and after (b) first, (c) second and (d) third precipitation. 

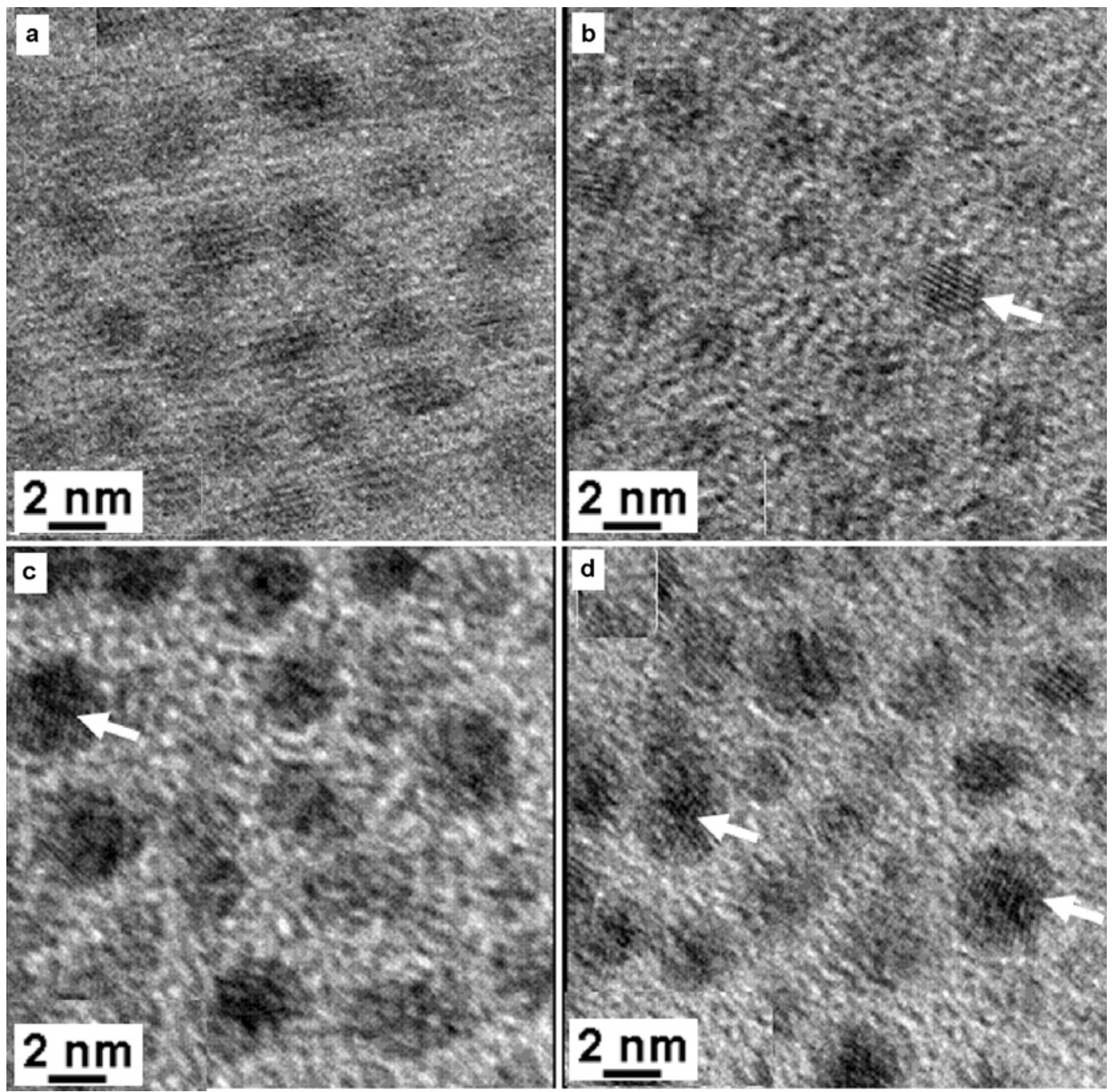

Figure 2. HRTEM images of Au@C18. (a) Before precipitation and after (b) first, (c) second and (d) third precipitation. The lattice fringes are marked with arrows. The distance between the particles is in the range of $1 \mathrm{~nm}$.

In the case of $\mathrm{Au}(\mathrm{C} 18$, all the materials showed peaks at positions matching with those of crystalline alkanes indicating that the alkyl chains are highly ordered. The $d^{t}$ peak for all the materials appeared at $2849 \mathrm{~cm}^{-1}$ and the $d^{+}$peak occurred at $2918 \mathrm{~cm}^{-1}$. From the first to the fourth sample, there was a gradual decrease in the intensity of the peak corresponding to the methyl symmetric stretching vibration. Figure 3B shows the region between 600 and $1300 \mathrm{~cm}^{-1}$. The most important observation was the appearance of well defined peaks between 1175 and $1260 \mathrm{~cm}^{-1}$. The bunch of peaks between 1100 and $1300 \mathrm{~cm}^{-1}$ is due to the progression bands arising out of the $\mathrm{CH}_{2}$ wagging mode. In the presence of a defect free all-trans alkane chain, the $\mathrm{CH}_{2}$ wagging mode couples to give such progression bands. The presence of sharp and well-defined progression bands between 1100 and $1300 \mathrm{~cm}^{-1}$ is a strong evidence for the crystallinity of the alkyl chains. As we go from traces $a$ to $c$, the wagging progression bands become more intense and clearly resolved. This normally happens when there is defect-free alkyl chain which allows the coupling of the methylene modes. The separation between the adjacent peaks in the progression series $(\Delta \gamma)$ allows us to calculate the number of all-trans methylenes in a polymethylene using the simple relation, $m+1=326 / \Delta \gamma$, where $m$ is the number of methylene units in the all-trans chain. ${ }^{18}$ The number of all-trans methylenes present in $\mathrm{Au} @ \mathrm{C} 18$ after second purification was 17, implying the presence of an almost defect-free alkyl chain. The $\mathrm{C}-\mathrm{S}$ stretching frequency can be used to probe 

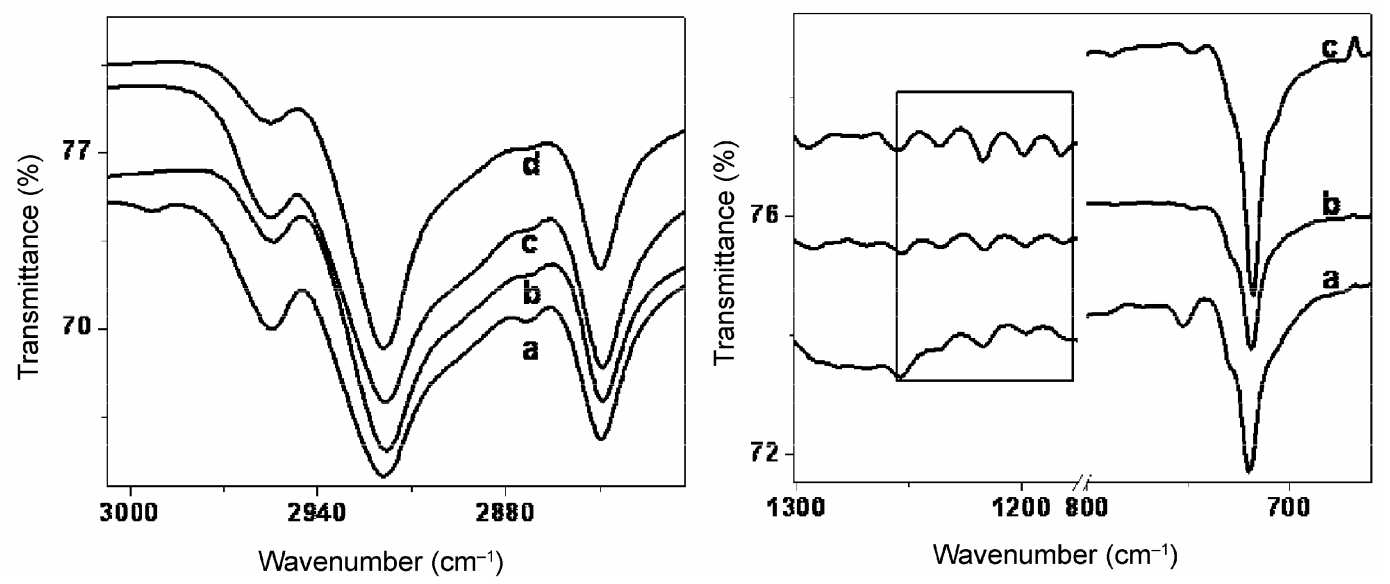

Figure 3. (A) IR spectra between 2700 and $300 \mathrm{~cm}^{-1}$ for $\mathrm{Au} @ \mathrm{C} 18$ after each stage of purification. (a) Before precipitation and after (b) first, (c) second and (d) third precipitations. (B) IR spectra between 600 and $1300 \mathrm{~cm}^{-1}$ for $\mathrm{Au}$ a C18 as a function of purification. (a) Before precipitation and after (b) first and (c) second precipitations. The square indicates the progression series arising from the $\mathrm{CH}_{2}$ waging mode.

the defects near the metal-organic interface. ${ }^{10}$ The IR spectrum normally shows two C-S peaks in the 600$750 \mathrm{~cm}^{-1}$ region, one corresponding to the gauche conformer $\left(v(C-S)^{G}\right)$ and the other due to the trans conformer $\left(v(C-S)^{T}\right)$. In the case of the octadecane thiol capped gold nanoparticles, the peak corresponding to the $\mathrm{C}-\mathrm{S}^{\mathrm{G}}$ conformer was absent in all the samples. Undetectable levels of gauche conformer indicates very little near-surface defects. The exact position of the $v(\mathrm{C}-\mathrm{S})^{\mathrm{T}}$ was difficult to judge as it merged with the methylene rocking mode $\left(\mathrm{P}_{\mathrm{x}}\right)$ at $720 \mathrm{~cm}^{-1}$.

The effect of PTR removal on the chain dynamics was studied by comparing the width of the carbon resonance in ${ }^{13} \mathrm{C}$ NMR after each stage of purification. Normally one would expect the width to decrease as the removal of PTR gives more space for the alkyl chain to exhibit dynamics. But to our surprise, the widths of the carbon resonances were found to increase upon repeated removal of the phase transfer agent. The analysis of the IR data in combination with the ${ }^{13} \mathrm{C}$ measurements indicates that the chains attain more order on repeated washing. Such relatively free chains in solution can bundle which results in restricted dynamics and broad NMR signals. The crystalline nature of the monolayer was very much clear from the infrared measurements. Figure 4 shows that the widths of all the carbon resonances increase on repeated removal of the phase transfer agent. Curve $a$ in figure 4A corresponds to the NMR of Au@ C18 taken before purification. Curve $b$ is after precipitating it once
$\mathrm{Au}$ a C18 from isopropyl alcohol/toluene mixture. The spectrum after precipitating twice is shown in curve $c$. The peak due to $\mathrm{C}_{2}$ carbon appeared at $39.25 \mathrm{ppm}$. The bunch of carbons from $\mathrm{C}_{3}$ to $\mathrm{C}_{15}$ appeared as a broad peak at $29 \mathrm{ppm}$. Additional peaks in trace $a$ of figure 4B are due to PTR. The peak corresponding to the terminal methyl carbon appeared at $14 \mathrm{ppm}$. Trace $a$ in figure $4 \mathrm{C}$ exhibits a split peak due to the presence of PTR. Of the two peaks in $a$, the peak at lower ppm is assigned to the PTR as it gets removed after precipitation. The peak due to carbon atoms adjacent to the thiol head group were absent in all the samples. The absence of carbon resonances close to the gold core indicates that they are in a dynamically restricted state.

We note that interdigitation which follows the PTR removal can also contribute to the reduced mobility of carbon atoms. As the PTR goes away form the nanoparticle surface, we expect more freedom for the rest of the alkyl chains to exhibit dynamics. Our studies on the dynamics of isolated clusters using neutron scattering measurements indicated that the dynamics evolves at a much lower temperature in isolated clusters compared to superlattices. ${ }^{12}$ This makes us to believe that in our NMR measurements, the dynamics of interdigitated structure is amplified, in contrast to isolated nanoparticles. Thus the experiments suggest that PTR removal in effect did not create room for the monolayers to exhibit dynamics. This is because interdigitation of the crystalline alkyl chains that followed the PTR removal made the carbon atoms away from the gold core to 


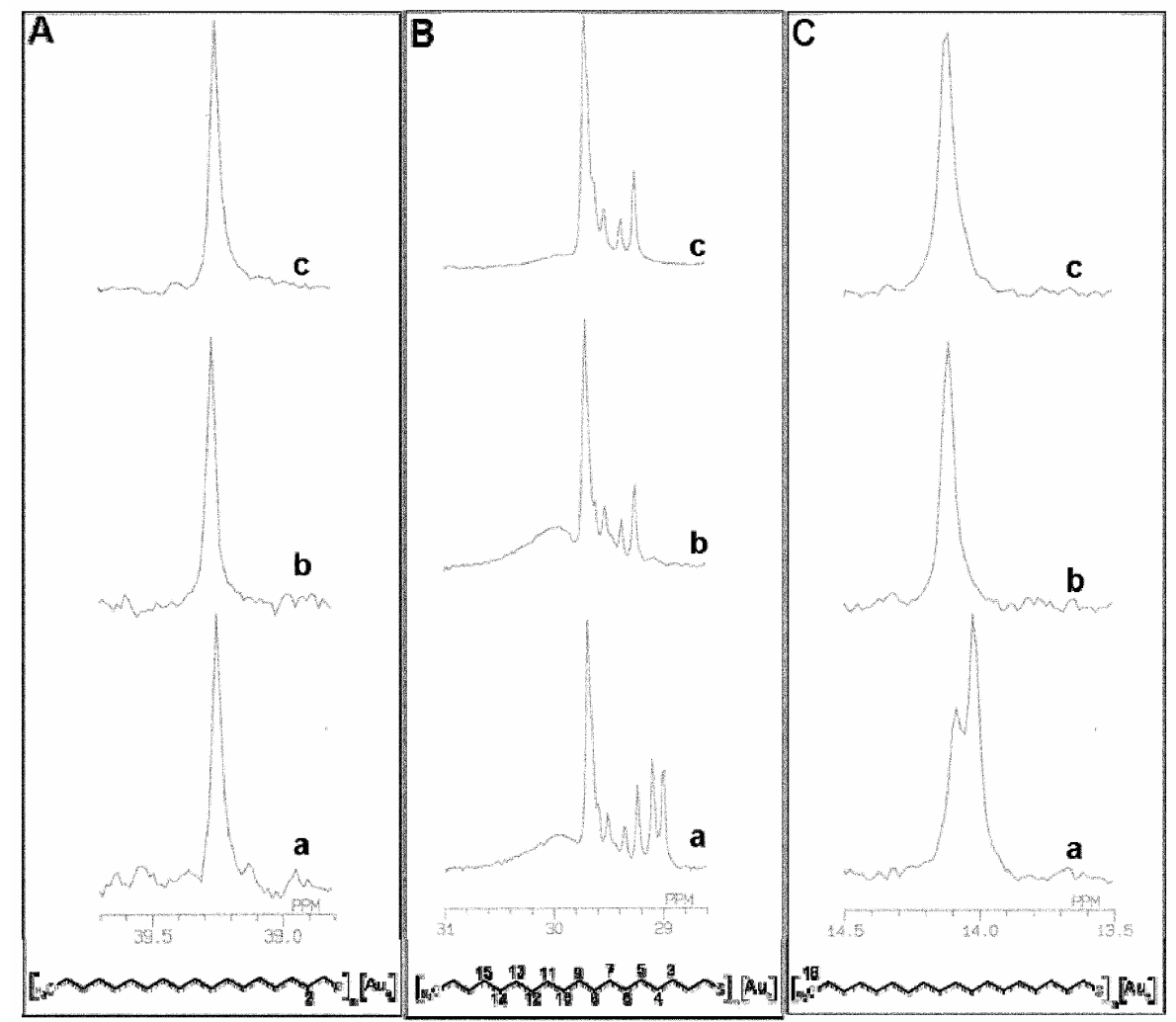

Figure 4. ${ }^{13} \mathrm{C}$ NMR spectra of $\mathrm{Au}$ (C18. The peak due to the carbon second to the thiol head group is shown in figure 4A. Curve (a) is before precipitation and after (b) first and (c) second precipitations. Each region of the spectrum is expanded to show the change in the width of the peak. The carbon resonances between $C_{3}$ and $C_{15}$ are shown in figure $4 \mathrm{~B}$. Figure $4 \mathrm{C}$ shows the peak due to the terminal methyl carbon. The structure of the thiol and the carbons responsible for the peaks are labelled.

pack themselves. Besides this, NMR measurements indicate that sample heterogeneity, significantly contributes to the dynamics observed in the system (this aspect is dealt with separately).

Figure 5A shows the ${ }^{1} \mathrm{H}$ NMR spectra of $\mathrm{Au}$ @) $\mathrm{C} 18$ taken at each stage of purification. Curve $a$ in figure 5A corresponds to the NMR of Au@ C18 taken after precipitating once from isopropyl alcohol/toluene mixture. Curve $b$ is after twice and curve $c$ is after thrice precipitation. The peak due to the carbon adjacent to the sulphur atom appeared at $1.55 \mathrm{ppm}$. The bunch of protons from $\mathrm{C}_{3}$ to $\mathrm{C}_{11}$ appeared as a singlet at $1.26 \mathrm{ppm}$. The peak corresponding to the terminal carbon appeared at $0.84 \mathrm{ppm}$. The protons of the carbon close to the gold core did not appear in the NMR spectrum. As expected, the carbons close to the gold core are solid-like with restricted mobility. This observation is in accordance with the earlier report. ${ }^{6}$ The carbons close to the gold core are in a dynamically restricted state which leads to large relaxation time. Hence the signal due to the protons attached to that carbon merges with the base line. The interdigitation also contributes to this effect. As we go from curve $a$ to curve $c$ in figure $5 \mathrm{~A}$, the width of the proton resonances increases. This indicates a reduced dynamics in the material with least amount of PTR. This also supports the interdigitation of alkyl chains. The effect of PTR removal was clear from the line-width of the methylene protons. In order to show the increase in peak width, the region between $1 \cdot 15-1.40 \mathrm{ppm}$ in the NMR spectrum of $\mathrm{Au} @ \mathrm{C} 18$ is shown in figure 5B.

Figure 6A shows the IR spectrum between 2700$3000 \mathrm{~cm}^{-1}$ for $\mathrm{Au} @ \mathrm{C} 12$. The curves labelled $a, b$ and $c$ show the IR spectra of $\mathrm{Au} @ \mathrm{C} 12$ taken before precipitation and after once, twice and thrice precipitating from isopropyl alcohol/toluene mixture. In the case of $\mathrm{Au} @ \mathrm{C} 12$, all the materials showed methylene peak at positions matching with that of the crystalline alkyl chain indicating that the alkyl chain is highly ordered. The $d^{+}$peak for all the materials appeared at $2850 \mathrm{~cm}^{-1}$ and the $d^{-}$peak occurred 
at $2919 \mathrm{~cm}^{-1}$. The IR spectra between 1000 and $1500 \mathrm{~cm}^{-1}$ region are shown in figure 6B. Literature reports the presence of two important peaks in this region; a peak corresponding to the methylene scissoring mode and another peak due to the $\mathrm{CH}_{3}$ symmetric bending vibration, known by the name, the umbrella mode. ${ }^{3}$ The region between 1300 $1400 \mathrm{~cm}^{-1}$ is useful in detecting the conformational disorder present in the alkane chain. The end gauche defect, if present, will show up as a peak at $1341 \mathrm{~cm}^{-1}$. The presence of peaks at 1307 and $1367 \mathrm{~cm}^{-1}$ indicates kinks in the alkane chain. The peak at $1354 \mathrm{~cm}^{-1}$ corresponds to double gauche. ${ }^{3}$ The absence of any of the above mentioned peaks indicates that the population of disorder present in the alkane chain is below the detection limit. The progression bands arising out of the wagging mode was much better resolved in the case of Au@ $\mathrm{C} 12$. As we go from one to four, wagging progression bands became very sharp. The presence of such sharp progression bands is an indication of crystalline alkyl chain. The number of all trans carbons calculated from the separation between the progression bands was 11 , implying that the alkyl chain is free of defects. The region between 600 and $1000 \mathrm{~cm}^{-1}$ in the IR spectrum of $\mathrm{Au}(\mathrm{C} 12$ is shown in figure 6C. A major peak at $720 \mathrm{~cm}^{-1}$, corresponding to methylene

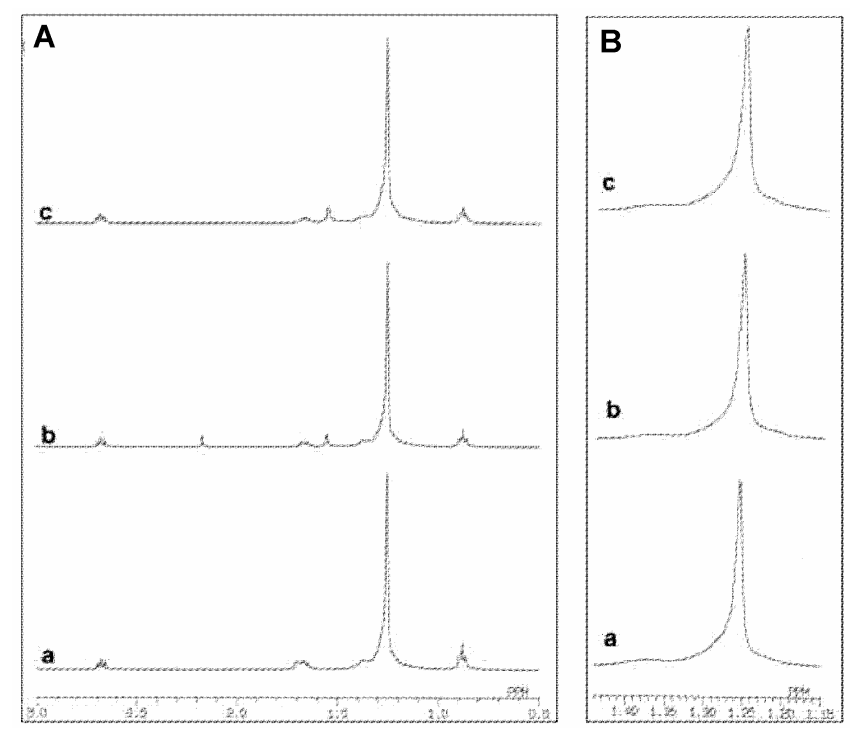

Figure 5A. ${ }^{1} \mathrm{H}$ NMR spectrum of $\mathrm{Au} @ \mathrm{C} 18$ taken after each stage of precipitation. Curve $a$ in figure $5 \mathrm{~A}$ is after first precipitation and after (b) second, (c) third precipitations. The region between $1 \cdot 15-1.40 \mathrm{ppm}$ is shown in figure $5 \mathbf{B}$. In figure $5 \mathbf{B}$, the line-width increases as we go from curve a to c. rocking mode $(\mathrm{Px})$, was present in all the samples along with the progression bands at 784, 783 and $828 \mathrm{~cm}^{-1}$. The rocking progression bands indicate the presence of considerable number of trans $\mathrm{CH}_{2}$ groups in an alkyl chain. The other features present in this region include the in-plane methyl rocking mode at $890 \mathrm{~cm}^{-1}$. Although the peak position was the same for all the materials, intensity varied from one to the other. The peak corresponding to the $(\mathrm{C}-\mathrm{S})^{\mathrm{G}}$ conformer was absent in all the samples. The undetectable levels of gauche conformer indicates very little near-surface defects. In a crystalline chain, the peak corresponding to $(\mathrm{C}-\mathrm{S})^{\mathrm{T}}$ will merge with the rocking mode, hence difficult to locate.

Figure 7 shows the ${ }^{1} \mathrm{H}$ NMR spectrum of $\mathrm{Au} @ \mathrm{C}_{12}$ at each stage of purification. Figure 7A is due to the protons attached to the second carbon atom from the thiol head group. Figure 7B is due to the carbon atoms from $\mathrm{C}_{3}-\mathrm{C}_{11}$. The peak due to the terminal methyl protons is shown in figure 7C. The peak due to $\mathrm{C}_{2}$ protons appeared at 1.55 (figure $7 \mathrm{~A}$ ). The bunch of protons from $\mathrm{C}_{3}$ to $\mathrm{C}_{11}$ appeared as a broad peak at $1.26 \mathrm{ppm}$ (figure 7B). The peak corresponding to the protons attached to the terminal carbon atom appeared at $0.84 \mathrm{ppm}$ (figure 7C). Curve $a$ in figure $7 \mathrm{~A}$ is after first precipitation, curve $b$ is after second precipitation and curve $c$ is taken after third precipitation from isopropyl alcohol/toluene mixture. In general, as we go from the first sample with large amount of PTR to the last sample with least amount of PTR, the width of the proton resonance increases. Trace $b$ in figure 7B is an exception where large increase in width is seen upon second purification. This indicates that the alkyl chain has little freedom to move as we go from the first sample to the last. By the repeated removal of the PTR, we expect a better dynamics for the alkyl chain. On the contrary, the chain mobility reduces on successive purification. As the PTR goes away from the monolayer, the chain attains order as revealed by infrared measurements. Such ordered all-trans alkyl chains have high propensity to form interdigitated structures. Thus the interdigitated alkyl chains are in a highly restricted environment, reducing the mobility of the alkyl chain and increasing the line width.

Another way to explain the line broadening is in terms of the adsorption sites available for the molecule upon the removal of the PTR. The faceted nanocrystal can provide adsorption sites with different energies. The different adsorption sites include edges, terraces or vertices. When there is PTR, all the adsorption sites may not be available to the thiol 

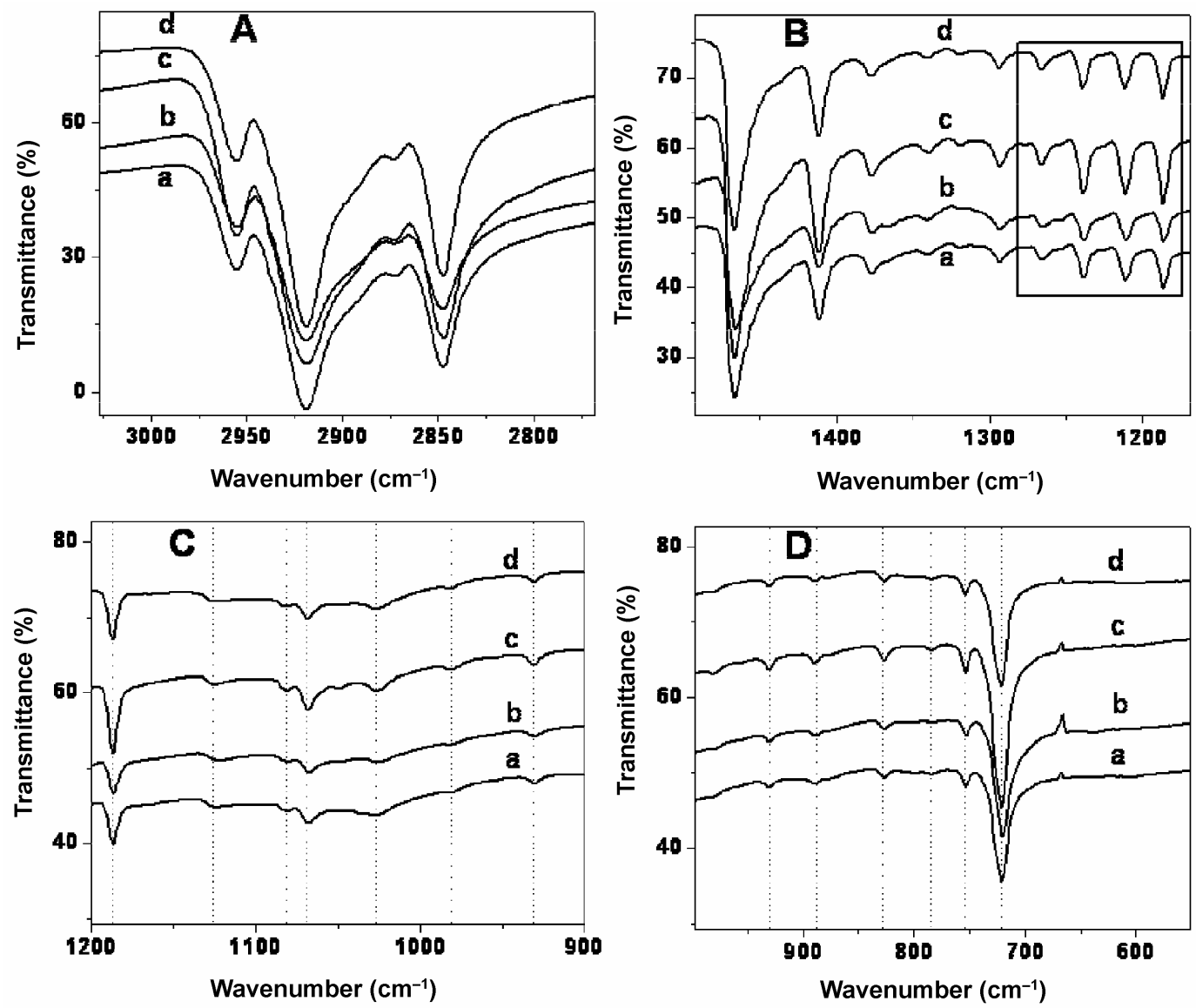

Figure 6. IR spectrum between (A) $2700-3000 \mathrm{~cm}^{-1}$, (B) $1100-1500 \mathrm{~cm}^{-1}$, (C) $900-$ $1200 \mathrm{~cm}^{-1}$ and (D) $550-1000 \mathrm{~cm}^{-1}$ for Au@C12. The rectangle indicates the progression series arising from the $\mathrm{CH}_{2}$ waging mode. (a) Before precipitation and after (b) first, (c) second and (d) third precipitation.
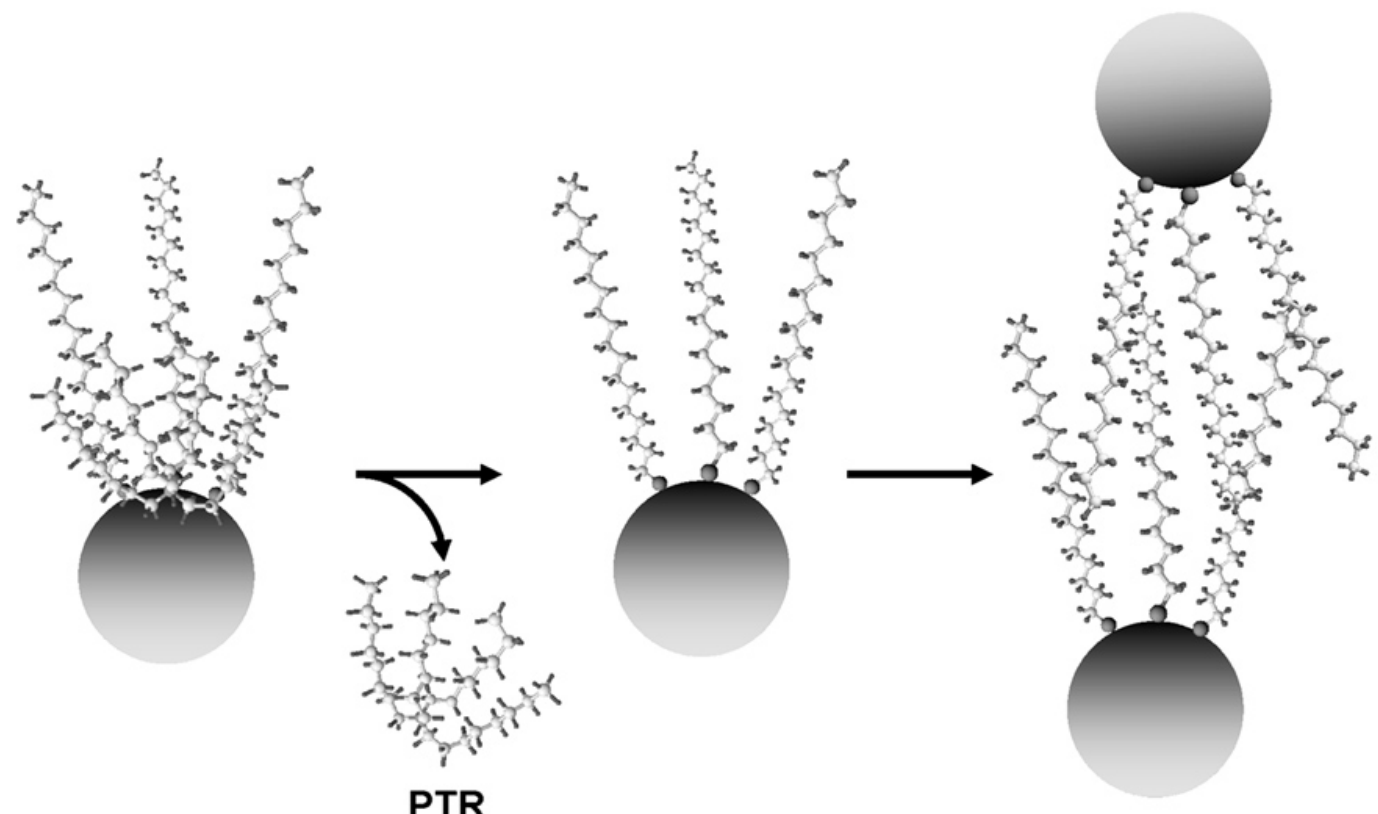

PTR

Scheme 1. Schematic showing the removal of the phase transfer agent, TOAB from the surface of gold nanoparticle and subsequent interdigitation of alkyl chains leading to restricted alkyl chain dynamics. 
head group. As the PTR is removed from the surface, the molecule will have access to all the different binding sites which creates a line broadening due to varying chemical shift values. ${ }^{10}$ This, however, does not support the IR data.

Thus the ${ }^{1}$ HNMR data show that the alkyl chains are in a dynamically restricted state even though the removal of PTR would imply that the monolayers would be more free to exhibit dynamics. The NMR measurements thus point to a reduced mobility brought about by the interdigitation of alkyl chains. A schematic showing the removal of PTR from the gold nanoparticle surface resulting in increased space for the alkyl chain to exhibit dynamics is given in

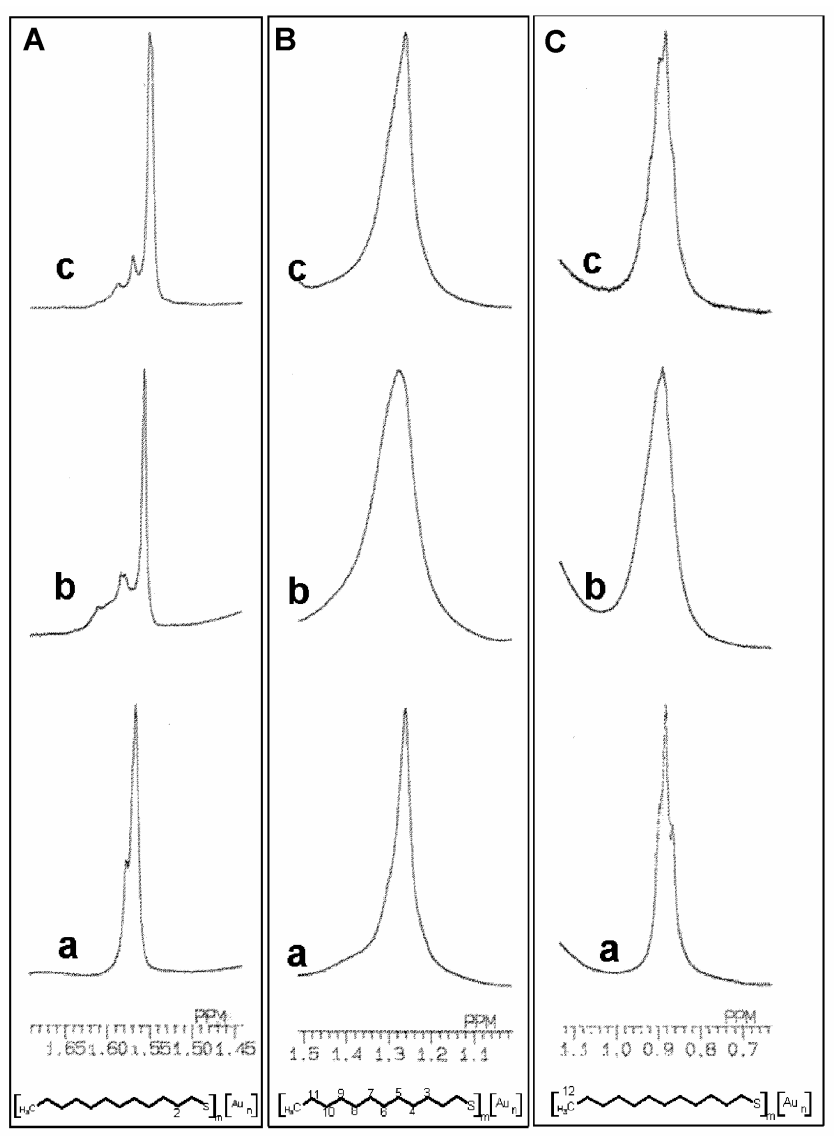

Figure 7. ${ }^{1} \mathrm{HNMR}$ spectra of $\mathrm{Au} @ \mathrm{C} 12$ after each stage of purification. Each region of the NMR spectrum is expanded to show the change in peak-width. The peak due to the protons attached to the second carbon from the thiol head group is shown in A. Curve (a) in $\mathbf{A}$ is the NMR spectrum taken after first precipitation, (b) and (c) are after second and third precipitation, respectively. The protons of carbon atoms between $\mathrm{C}_{3}$ and $\mathrm{C}_{11}$ are shown in B. C shows the peak due to the terminal methyl protons. The structure of the thiol and the methylenes/methyl responsible for the peaks are labelled. scheme 1. The presence of PTR prevents the chains from executing dynamics. The monolayers after the PTR removal have enough room available for exhibiting motions as shown in scheme 1. Although we could succeed in removing the PTR to a considerable extent, the interdigitation of alkyl chains that followed the removal prevented us from observing the dynamics expected for an all-trans alkyl chain.

It is important to keep in mind a few additional details while making further suggestions. The nanoparticles are faceted and as a result, the alkyl chains form bundles on these crystal planes. ${ }^{13,19}$ The bundles themselves have reduced dynamics, which may be reflected in the measurements. The bundling happens as a result of the removal of PTR. The presence of different types of bundles, formed on different planes imply that the methylenes can be in different chemical environments, which contribute to differences in chemical shifts, leading to peak broadening. The existence of aggregates, as revealed in the $\mathrm{UV} / \mathrm{Vis}$ measurements suggests that the chains of adjacent nanoparticles are interdigitating. Such aggregate formation can reduce the dynamics. Thus the observed effects could be a composite of all these; but the reduced dynamics due to the removal of PTR is distinctly clear.

\section{Conclusions}

The repeated purification of thiol protected gold nanoparticles resulted in a defect-free alkyl chain. The presence of such ordered all-trans alkyl chain was confirmed by the IR spectroscopy. ${ }^{1} \mathrm{H}$ and ${ }^{13} \mathrm{C}$ NMR measurements as a function of purification indicated reduction in the mobility of alkyl chains even though repeated purification results in the removal of the phase transfer reagent, creating more room for the alkyl chains to exhibit dynamics. The reduced mobility observed in the system is explained in terms of the restriction brought about by the interdigitation of alkyl chains. The signatures of such interdigitated particles were seen in UV-Vis, IR and TEM measurements. We are aware that there are additional complexities in attributing the peakwidth to dynamics alone. Repeated removal of the phase transfer agent may create new adsorption sites, which were not accessible to the thiol head group previously. Thus the observed line broadening is a complex phenomenon involving multiple factors such as sample heterogeneity, dynamics and availability of adsorption sites. 


\section{Acknowledgements}

T P thanks Department of Science and Technology (DST), India for supporting research programme on nanomaterials. RKVR is supported by a research grant from (DST).

\section{References}

1. Brust M, Walker M, Bethell D, Schiffrin D J and Wyman R 1994 Chem. Commun. 801

2. Templeton A C, Wuelfing W P and Murray R W 2000 Acc. Chem. Res. 3327

3. Hostetler M J, Stokes J J and Murray R W 1996 Langmuir 123604

4. Hostetler M J, Green S J, Stokes J J and Murray R W 1996 J. Am. Chem. Soc. 1184212

5. Terril R H, Postlethwaite T A, Chen C H, Poon C D, Terzis A, Chen A, Hutchison J E, Clark M R, Wignall G, Londono J D, Superfine R, Falvo M, Johnson C S, Samulski E T and Murray R W $1995 \mathrm{~J}$. Am. Chem. Soc. 11712537

6. Badia A, Gao W, Singh S, Demers L, Cuccia L and Reven L 1996 Langmuir 121262

7. Schmitt H, Badia A, Dickinson L, Reven L and Lennox R B $1998 A d v$. Mater. 10475

8. Badia A, Cuccia L, Demers L, Morin F and Lennox R B 1997 J. Am. Chem. Soc. 1192682

9. Mar W and Klein M L 1994 Langmuir 10188
10. Hostetler M J, Wingate J E, Zhong C J, Harris J E, Vachet R W, Clark M R, Londono J D, Green S J, Stokes J J, Wignall G D, Glish G L, Porter M D, Evans N D and Murray R W 1998 Langmuir 1417

11. Mukhopadhyay R, Mitra S, Pradeep T, Tsukushi I and Ikeda S 2003 J. Chem. Phys. 1184614

12. Mitra S, Nair B, Pradeep T, Goyal P S and Mukhopadhyay R 2002 J. Phys. Chem. B106 3960

13. Sandhyarani N, Resmi $M$ R, Unnikrishnan R, Vidyasagar K, Ma S, Antony M P, Selvam G P, Visalakshi V, Chandrakumar N, Pandian K, Tao Y T and Pradeep T 2000 Chem. Mater. 12104

14. Mukhopadhyay R, Mitra S, Johnson M, Kumar V R R and Pradeep T 2007 Phys. Rev. B75 75414

15. Prasad B L V, Stoeva S I, Sorensen C M and Klabunde K J 2002 Langmuir 187515

16. Sandhyayrani N and Pradeep T 2003 Int. Rev. Phys. Chem. 22221

17. Badia A, Singh S, Demers L, Cuccia L, Brown G R and Lennox R B 1996 Chem. Eur. J. 2359

18. (a) Snyder R G 1967 J. Chem. Phys. 47 1316; (b) Snyder R G, Maroncelli M, Qi S P and Strauss H L 1981 Science 214 188; (c) Maroncelli M, Qi S P, Strauss H L and Snyder R G $1982 \mathrm{~J}$. Am. Chem. Soc. 104 6237; (d) Hagemann H, Strauss H L and Snyder R G 1987 Macromolecules 20 2810; (e) Krimm S 1960 Adv. Polym. Sci. 2 51; (f) Snyder R G $1961 \mathrm{~J}$. Mol. Spectrosc. 7 116; (g) Snyder R G and Schachtschneider J H 1963 Spectrochim. Acta A19 117

19. Sandhyarani N, Pradeep T, Chakrabarti J, Yousuf M and Sahu H K 2000 Phys. Rev. B62 739 